\title{
Tethered spinal cord syndrome detected during ultrasound for caudal block in a child with single urological anomaly
}

\author{
Jeongmin Kim ${ }^{1,2}$, Seokyung Shin ${ }^{1,2}$, Hyein Lee ${ }^{1}$, and Hae Keum Kil ${ }^{1,2}$ \\ ${ }^{1}$ Department of Anesthesiology and Pain Medicine, ${ }^{2}$ Anesthesia and Pain Research Institute, Yonsei University College of Medicine,
} Seoul, Korea

With the increasing use of ultrasound for assuring safe and accurate caudal or epidural block for postoperative analgesia in children, hidden spinal dysraphism may be found incidentally. Children with congenital anomalies such as VATER or VACTERL frequently present with accompanying occult spinal dysraphisms (OSD), which is characterized with skincovered lesions such as tethered spinal cord (TSC), meningocele, or lipomyelomeningocele. OSDs are usually found incidentally during the evaluation for other diseases or after neurological symptoms manifest. However, in children with a simple urogenital anomaly, OSD may not be found until the symptoms of OSD manifest because ultrasound screening is not routinely performed in neonates. We report a case of TSC that was discovered during routine ultrasound for caudal block in an asymptomatic child undergoing urological surgery. A 12-month-old, $9.8 \mathrm{~kg}$, otherwise healthy female infant was
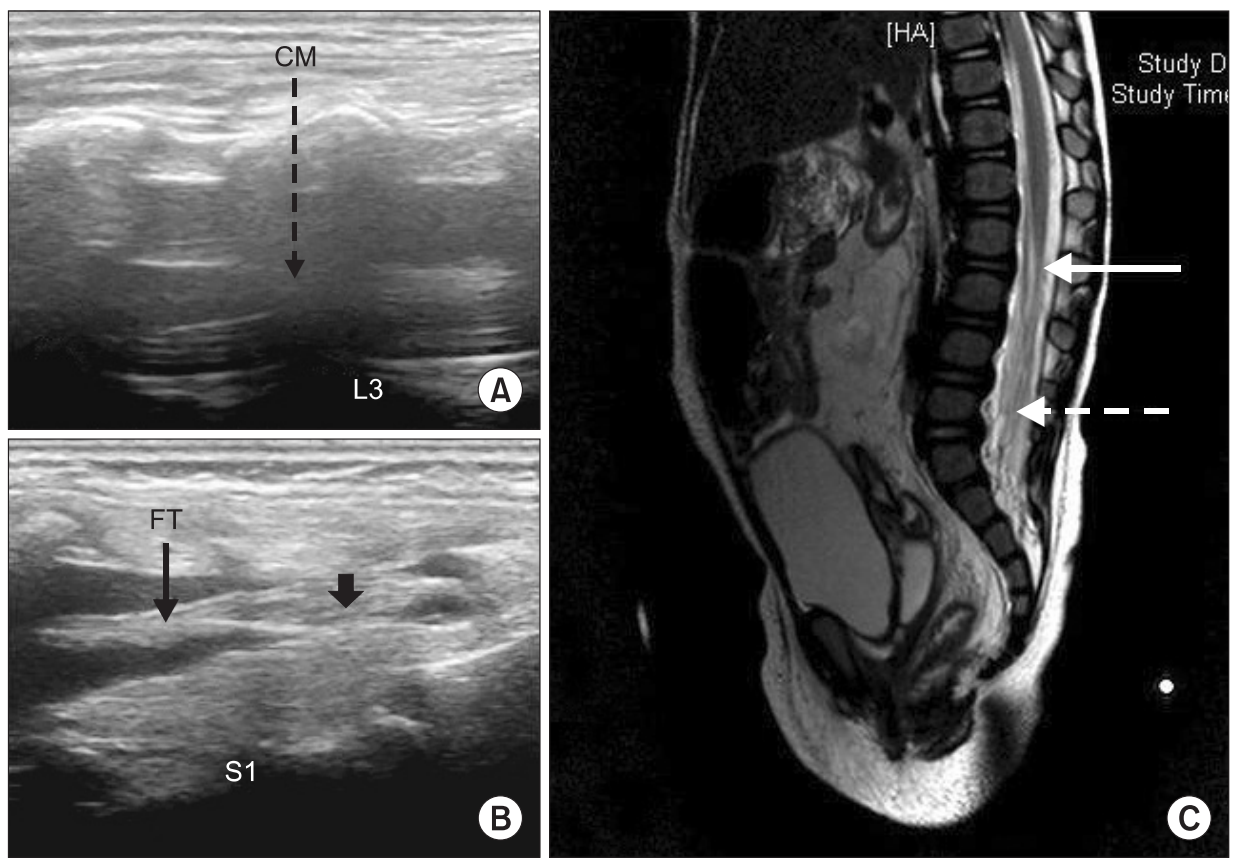

Fig. 1. Ultrasound (A, B) and MRI (C) findings. CM: conus medullaris, FT: filum terminale. In (C), the solid arrow and dotted arrow indicate the low-lying $\mathrm{CM}$ and thickened FT.

Corresponding author: Hae Keum Kil, M.D., Department of Anesthesiology and Pain Medicine, and Anesthesia and Pain Research Institute, Yonsei University College of Medicine, 50, Yonsei-ro, Seodaemun-gu, Seoul 120-752, Korea. Tel: 82-2-2228-2420, Fax: 82-2-312-7185, E-mail: hkkil@yuhs.ac

(c) This is an open-access article distributed under the terms of the Creative Commons Attribution Non-Commercial License (http:// creativecommons.org/licenses/by-nc/3.0/), which permits unrestricted non-commercial use, distribution, and reproduction in any medium, provided the original work is properly cited. 
scheduled to receive a pyeloureterostomy for hydronephrosis. When the patient was placed in lateral decubitus position for caudal block after general anesthesia, an intergluteal simple dimple (1.2 cm in length) was noted. Upon ultrasound (LOGIQe $^{\mathrm{TM}}$; GE Healthcare, Wauwatosa, WI, USA) for the spinal structure, we found a thickened filum terminale (FT) $2.2 \mathrm{~mm}$ in diameter with low-lying conus medullaris (CM) (L3 level) (Fig. 1A and 1B). We did not perform a caudal block and recommended magnetic resonance imaging (MRI) study for further evaluation. In the postoperative MRI, TSC with FT lipoma was confirmed (Fig. 1C) and detethering surgery was performed after one month.

The prognosis of TSC depends mostly on early diagnosis and treatment. While the exact incidence of TSC is not known, greater clinical awareness and MRI diagnostics have lead to a higher rate of incidental detection. Although several studies have reported that children with lumbosacral lipoma have other congenital malformations combined with urogenital anomalies, the association between TSC with a single urogenital anomaly has not been clearly established [1,2]. There have been arguments that minor skin markers are not a stigmata of OSD. One study found OSD to be present in children with minor skin markers and in children without skin markers [3]. Compared to normal infants, in which the CM terminates above $\mathrm{Ll} / 2$ and the thickness of the FT is $<2 \mathrm{~mm}$ at the L5/S1 level, the CM in this patient was located at the L3 level and the FT thickness was 2.2 mm upon ultrasound. Therefore, a pediatric patient presenting with a single urogenital anomaly together with a minor skin marker may require greater attention with further investigation into a possible underlying OSD.

Ultrasound is a simple and effective screening method for detecting spinal abnormalities. In clinical practice, if the ultrasound is available for a caudal block, lumbosacral spinal structures should be evaluated thoroughly even in children with a single urogenital anomaly.

\section{References}

1. Pierre-Kahn A, Zerah M, Renier D, Cinalli G, Sainte-Rose C, LellouchTubiana A, et al. Congenital lumbosacral lipomas. Childs Nerv Syst 1997; 13: 298-334

2. Scheible W, James HE, Leopold GR, Hilton SV. Occult spinal dysraphism in infants: screening with high-resolution real-time ultrasound. Radiology 1983; 146: 743-6.

3. Henriques JG, Pianetti G, Henriques KS, Costa P, Gusmao S. Minor skin lesions as markers of occult spinal dysraphisms--prospective study. Surg Neurol 2005; 63: S8-S12. 